\title{
Strongly reduced bias dependence in spin-tunnel junctions obtained by ultraviolet light assisted oxidation
}

\author{
H. Boeve ${ }^{a)}$ \\ Kapeldreef 75, B-3001 Leuven, Belgium \\ E. Girgis and J. Schelten \\ Institute for Thin Film and Ion Technology, Research Center Jülich GmbH, D-52425 Jülich, Germany \\ J. De Boeck and G. Borghs \\ Kapeldreef 75, B-3001 Leuven, Belgium
}

(Received 9 September 1999; accepted for publication 21 December 1999)

For future implementation of ferromagnetic tunnel junctions, we need a better understanding of the influence of the insulating barrier preparation method on the junction resistance, tunnel magnetoresistance (TMR), and its voltage bias dependence. In this letter, we focus on the bias dependence of junctions $\left(\mathrm{Co}-\mathrm{Al}_{2} \mathrm{O}_{3}-\mathrm{Ni}_{80} \mathrm{Fe}_{20}\right)$ prepared by ultraviolet light assisted in situ oxidation in an $\mathrm{O}_{2}$ ambient. For an initial Al thickness of $1.3 \mathrm{~nm}$, the resistance times area product of the junctions is $60 \mathrm{k} \Omega \mu \mathrm{m}^{2}$, while showing up to $20 \%$ TMR at $5 \mathrm{mV}$ bias. The decrease of TMR with bias voltage up to $1 \mathrm{~V}$ is remarkably small leading to $V_{1 / 2}$, for which half of the low-bias TMR remains, well over 0.6 V. (C) 2000 American Institute of Physics. [S0003-6951(00)02908-9]

Tunnel-type magnetoresistance (TMR) occurring in devices, which consist of two ferromagnetic films separated by an insulating layer, has received great attention in recent years. In these tunnel-type magnetic nanostructures, the charge transport is governed by tunneling through the insulating layer, generally produced by sputter deposition of a thin aluminum layer followed by oxidation. The electron tunneling current depends on the relative orientation of the magnetic moments in the two ferromagnetic films. More exactly, it is directly proportional to the polarization values of the electrons at the two insulator/metal interfaces, which may be different from the bulk polarization of the two magnetic films.

For the future implementation of ferromagnetic tunnel junctions in practical applications, such as read heads and magnetoresistive random access memories, all characteristics of the tunnel devices have to be critically controlled. The small device size required by the applications leads to the need of small resistance-area (RA) products of 1-10 $\mathrm{k} \Omega \mu \mathrm{m}^{2}$, in order to keep the device resistance and $\mathrm{RC}$ time constant at an acceptable level. Another important parameter in the tunneling process between the two magnetic films is the bias voltage over the insulating layer., ${ }^{2,3}$ The bias voltage dependence of the TMR may convey information about the detailed process of spin-polarized tunneling. ${ }^{4}$

In general, we need a more complete understanding of the relationships between the resistance, the low-bias TMR, and the reliability of the junctions measured by the breakdown voltage of the junctions. The preparation of the insulator barrier is key in the behavior of these characteristics. In this letter we focus on the excellent bias voltage dependence of junctions prepared by ultraviolet light assisted in situ oxidation in an $\mathrm{O}_{2}$ ambient.

Ferromagnet-insulator-ferromagnet junctions were fab-

a)Electronic mail: boeve@imec.be ricated on (100)-oriented 4-in. silicon wafers capped with a $200 \mathrm{~nm}$ thermally grown $\mathrm{SiO}_{2}$. The top and the bottom ferromagnetic layers consist of either Co or $\mathrm{Ni}_{80} \mathrm{Fe}_{20}$. After deposition of the bottom electrode using dc magnetron sputtering in an $\mathrm{Ar}$ ambient of $6 \times 10^{-3}$ mbar, the aluminum layer with a thickness of $1.3 \mathrm{~nm}$ was produced using rf sputtering in $2 \times 10^{-2}$ mbar, followed by an in situ oxidation for an hour using an ultraviolet lamp in the chamber in which 100 mbar of high purity oxygen $\left(\mathrm{O}_{2}\right)$ was maintained. ${ }^{5,6}$ After the oxidation process the top electrode was sputtered using dc magnetron sputtering. The junctions were patterned by optical lithography and ion beam etching. The bottom electrode was defined in the first mask level while the second resist mask patterns the top electrode and the junction. Contact openings to the top electrode are achieved in a selfaligned way by deposition of a $100 \mathrm{~nm} \mathrm{SiO}_{2}$ and liftoff of the resist etch mask on top of the junction. The junction area is scaling from 4 to $600 \mu \mathrm{m}^{2}$. The junctions are then contacted by depositing $10 \mathrm{~nm} \mathrm{TiW/160} \mathrm{nm} \mathrm{Au}{ }^{7}$

The $I(V)$ and $d I / d V(V)$ characteristics of a 3 by $15 \mu \mathrm{m}^{2}$ tunnel junction patterned into the layer structure $\mathrm{Si} / \mathrm{SiO}_{2} / 18$ $\mathrm{nm} \mathrm{Co} / 1.3 \mathrm{~nm} \mathrm{Al}$ (oxidized)/18 $\mathrm{nm} \mathrm{Ni} \mathrm{N}_{80} \mathrm{Fe}_{20}$, see Fig. 1, reveal a nonlinear behavior that is in good agreement with theoretical $I(V)$ curves by Simmons' model. ${ }^{8}$ Typical fit parameters obtained are an effective barrier height $\phi=2.8 \mathrm{eV}$ and an effective barrier thickness $d=0.9 \mathrm{~nm}$. The nonlinearity is caused by a decrease of the effective barrier height with increasing applied voltage. The RA product is $60 \mathrm{k} \Omega \mu \mathrm{m}^{2}$. Breakdown voltages are above $1.5 \mathrm{~V}$ which indicates an excellent dielectric quality of the insulating layer formed by ultraviolet light assisted oxidation which allows us to measure the magnetoresistance properties up to $1 \mathrm{~V}$, reproducibly and repeatedly, without breakdown occurring.

The magnetoresistance curves of a $10 \mu \mathrm{m}$ by $6 \mu \mathrm{m}$ tunnel junction in Fig. 2 reveal two different states with either parallel (low resistance) or antiparallel (high resistance) 


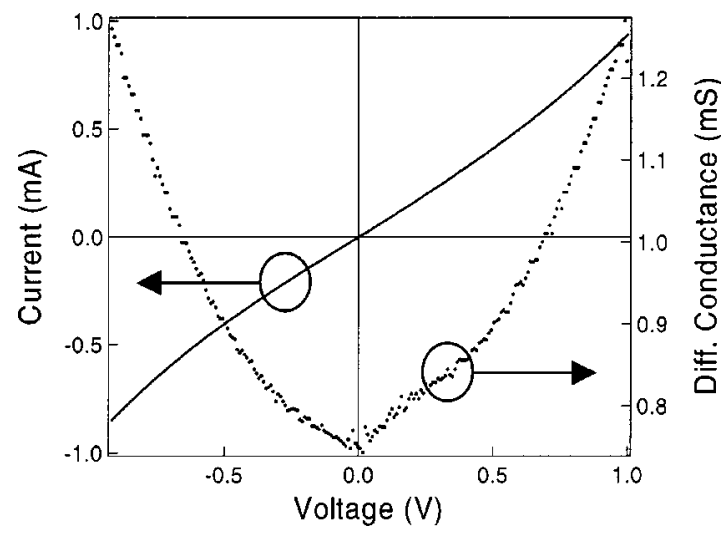

FIG. 1. $I(V)$ and calculated $d I / d V(V)$ characteristics for a $45 \mu \mathrm{m}^{2}$ junction $18 \mathrm{~nm} \mathrm{Co} / 1.3 \mathrm{~nm} \mathrm{Al}$ (oxidized) $/ 18 \mathrm{~nm} \mathrm{Ni} \mathrm{Ni}_{80} \mathrm{Fe}_{20}$. Typical fit parameters obtained from Simmons' model (Ref. 8) are $\theta=2.8 \mathrm{eV}$ for the barrier height and $d=0.9 \mathrm{~nm}$ for the barrier thickness.

alignment of the magnetization vectors in the ferromagnetic layers. The measured resistance difference between both states leads to a TMR value of $17 \%$ at low bias $(5 \mathrm{mV})$. When measured at higher bias voltages, the relative TMR value decreases. The shape of the magnetoresistance curves is identical, as the increased current density in the vertical tunnel device does not influence the magnetic properties. Starting from positive magnetic fields and continuing for small negative fields, a rather slow resistance increase can be observed when the soft $\mathrm{Ni}_{80} \mathrm{Fe}_{20}$ top electrode is switching. Given the absence of an induced magnetocrystalline anisotropy in the ferromagnetic layers, the magnetoresistance curves are set by domain effects during the switching process of the $\mathrm{Ni}_{80} \mathrm{Fe}_{20}$ top electrode. The sharp resistance changes at \pm 50 Oe correspond with the switching field of the large Co bottom electrode. By increasing of the aspect ratio of the tunnel junction, thus the top electrode, the shape anisotropy influences the switching behavior of the top electrode that results in a further increase in TMR and better defined plateaus in the magnetoresistance curve. A maximum TMR of $20 \%$ was observed in $20 \mu \mathrm{m}$ by $3 \mu \mathrm{m}$ tunnel junctions.

Also shown in Fig. 2 are the magnetoresistance curves measured at a voltage level of $0.5 \mathrm{~V}$ and $1 \mathrm{~V}$ using constant current bias. The low-bias TMR ratio of $17 \%$ at $5 \mathrm{mV}$ decreases to $11.2 \%$ at $500 \mathrm{mV}$ and $7 \%$ at $1 \mathrm{~V}$. The voltage $V_{1 / 2}$, at which the TMR is reduced by $50 \%$, is around 800

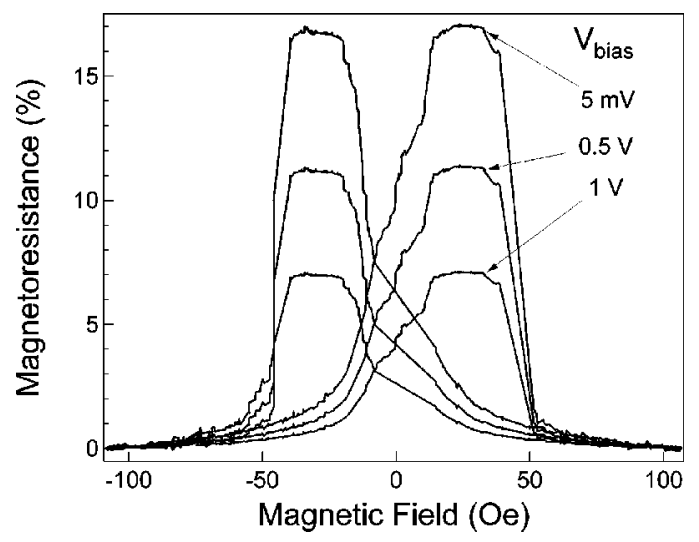

FIG. 2. Magnetoresistance curves for a 6 by $10 \mu \mathrm{m}^{2}$ junction showing $17 \%$ TMR at $5 \mathrm{mV}, 11.2 \%$ at $500 \mathrm{mV}$, and $7 \%$ at $1 \mathrm{~V}$ voltage bias. The layer

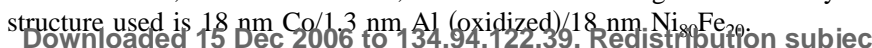

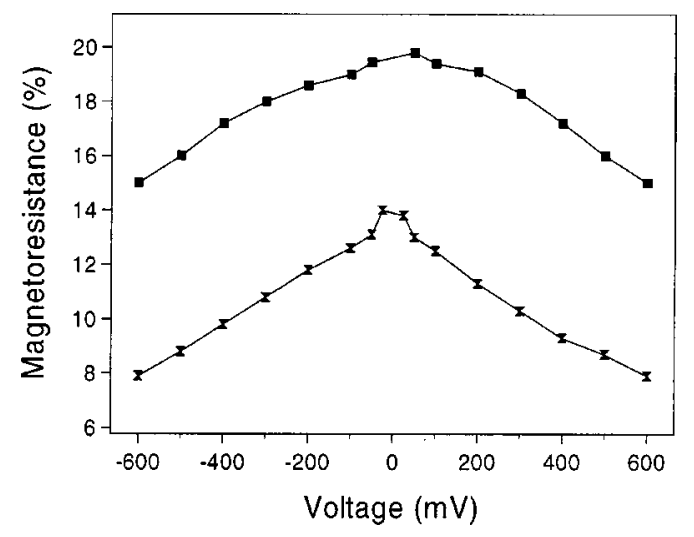

FIG. 3. Symmetrical bias dependence up to $0.6 \mathrm{~V}$ for two junctions with different low-bias TMR. Both curves show the same decay of $1 \%$ per 100 $\mathrm{mV}$. This trend has been observed in all processed junctions, irrespective of the absolute low-bias TMR value.

$\mathrm{mV}$ and is significantly higher than previously reported values for other postoxidation techniques. Starting from the same Al thickness, natural oxidation in situ or in air gives tunnel junctions with a lower RA product as well as lower TMR values, ${ }^{9}$ compared to our ultraviolet light assisted oxidation process. On the other hand an optimized plasma oxidation technique can give similar RA products but superior low-bias TMR values. ${ }^{10,11}$ Nonetheless, the bias dependence for both methods looks similar and gives voltages $V_{1 / 2}$ from $0.3 \mathrm{~V}$ for natural oxidation to $0.45 \mathrm{~V}$ for plasma oxidation. We believe that the observed improvement in bias dependence originates from the oxidation process itself. In our ultraviolet light assisted oxidation, a more reactive environment is created by the generation of ozone. The oxidation rate is increased with respect to natural oxidation and therefore, during oxidation, a better barrier uniformity can be obtained. The reduced oxidation time and presence of ozone limits any possible barrier contamination during the oxidation, e.g., due to carbon. Moreover, damage or defect generation due to bombardment of high energetic species as in plasma oxidation processes is avoided. Nonuniformities and defects are known to deteriorate the tunnel junction bias dependence by an increased contribution of spin-independent two-step tunneling, or other spin-flip processes. ${ }^{12,13}$

In our samples we have found that over $80 \%$ of the junctions, processed over many wafers, have low-bias TMR values higher than $10 \%$. All magnetic tunnel junctions, irrespective of their low-bias TMR values, show the same trends with respect to the bias dependence. In Fig. 3, the voltage bias dependence of the TMR is shown from low-bias up to $\pm 0.6 \mathrm{~V}$ as composed from several full magnetoresistance curves of two tunnel junctions with a different low-bias TMR. The bias dependence is symmetrical for positive and negative applied bias voltages. Both junctions have the same area $\left(60 \mu \mathrm{m}^{2}\right)$ and resistance $(1 \mathrm{k} \Omega)$ but a different aspect ratio, $20 \mu \mathrm{m}$ by $3 \mu \mathrm{m}$ and $10 \mu \mathrm{m}$ by $6 \mu \mathrm{m}$, which causes a difference in their low-bias TMR, $20 \%$ and $14 \%$, respectively. Notice that for both devices, at $\pm 0.6 \mathrm{~V}$, the remaining TMR signal is still over half of the original low-bias value (see Fig. 3; TMR at $\pm 0.6 \mathrm{~V}$ is $15 \%$ and $8 \%$, respectively). More importantly, both devices show the same decay of approximately $1 \%$ per $100 \mathrm{mV}$, a trend which can also be seen in the voltage dependence of the magnetoresistance curves of o AIP license or copyright, see http://apl.aip.org/apl/copyright.jsp 
Fig. 2. Therefore, a further increase of the modest TMR values measured in our junctions gives a good perspective on further improvement.

In conclusion, we presented the reduced bias voltage dependence of high quality ferromagnetic tunnel junctions by magnetron sputtering of $\mathrm{Al}$ followed by an ultraviolet light assisted oxidation process. The devices were patterned using optical lithography and have a RA product of $60 \mathrm{k} \Omega \mu \mathrm{m}^{2}$. The maximum low-bias magnetoresistance ratio is $20 \%$ for a $20 \mu \mathrm{m}$ by $3 \mu \mathrm{m}$ junction. The bias dependence was explored up to $1 \mathrm{~V}$ and its uniqueness is demonstrated: a constant $1 \%$ per $100 \mathrm{mV}$ decay, leading to voltages $V_{1 / 2}$ well over $0.6 \mathrm{~V}$. The small bias dependence in tunnel junctions fabricated using this oxidation method is an important advantage for practical applications, relaxing the operation voltage conditions of TMR elements.

The authors wish to thank P. Rottländer, H. Kohlstedt, and P. Grünberg for discussion and S. Peeters for help in processing. This work was partly supported by the European community ESPRIT Research project No. 20.027 (NM)2.
H.B. wishes to thank the IWT-Flanders for the financial support in the form of a scholarship.

${ }^{1}$ J. S. Moodera, L. R. Kinder, T. M. Wong, and R. Meservey, Phys. Rev. Lett. 74, 3273 (1995).

${ }^{2}$ T. Lu, X. W. Li, G. Q. Gong, G. Xiao, A. Gupta, P. Lecoevre, J. Z. Sun, Y. Y. Wang, and V. P. David, Phys. Rev. B 54, R15 629 (1996).

${ }^{3}$ S. Kumagai, T. Yaoi, and T. Miyazaki, J. Magn. Magn. Mater. 166, 71 (1997).

${ }^{4}$ J. S. Moodera and L. R. Kinder, J. Appl. Phys. 79, 4724 (1996); J. S. Moodera, J. Novak, and R. J. M. van de Veerdonk, Phys. Rev. Lett. 80, 2941 (1998).

${ }^{5}$ E. Girgis, J. Schelten, P. Grünberg, P. Rottländer, and H. Kohlstedt, IEEE Electron Device Lett. (to be published).

${ }^{6}$ I. W. Boyd, V. Craciun, and A. Kazor, Jpn. J. Appl. Phys., Part 1 32, 6141 (1993).

${ }^{7}$ W. J. Gallagher, S. S. P. Parkin, Y. Lu, X. P. Bian, A. C. Marley, K. P. Roche, R. A. Altman, S. A. Rishton, C. Jahnes, T. M. Shaw, and G. Xiao, J. Appl. Phys. 81, 3741 (1997).

${ }^{8}$ J. G. Simmons, J. Appl. Phys. 34, 1793 (1963).

${ }^{9}$ M. Sato and K. Kobayashi, IEEE Trans. Magn. 33, 3553 (1997).

${ }^{10}$ J. J. Sun and P. P. Freitas, J. Appl. Phys. 85, 5264 (1999).

${ }^{11}$ Y. Lu, X. W. Li, G. Xiao, R. A. Altman, W. J. Gallagher, A. Marely, K. Roche, and S. S. P. Parkin, J. Appl. Phys. 83, 6515 (1998).

${ }^{12}$ J. Zhang and R. M. White, J. Appl. Phys. 83, 6512 (1998).

${ }^{13}$ A. M. Bratkovsky, Appl. Phys. Lett. 72, 2334 (1998). 